\title{
CPI Prediction Based on ARIMA Model
}

\author{
Xiao Zhang \\ Institute of economics, Shanghai University, Shanghai, China \\ Eiffel_zx@163.com
}

Keywords: CPI prediction; ARIMA model

\begin{abstract}
In recent years, with the increasing attention of CPI, the academic research results on CPI prediction model emerge in endlessly. So far, the forecasting model about CPI in China mainly focus on the following aspects: time series forecasting model and VAR forecasting model, grey prediction model, regression forecast model and exponential smoothing model, neural network.

This paper mainly uses the monthly CPI data from January 2008 to December 2016, and describes the internal driving mechanism of CPI based on the ARIMA model, and predicts CPI on this basis.
\end{abstract}

\section{An Introduction to the ARIMA Model and the CPI Prediction}

The consumer price index (CPI) is an important indicator of macroeconomic analysis and decision-making, general price level detection and regulation, and national economic accounting. It is not only an important indicator of the extent of inflation, but also a reduction indicator in the national economic accounting.

It is generally assumed that a random sequence consists of a linear combination of random shocks, and can be described by a general linear model. ARIMA model is a lack of theoretical model, it does not need to rely on economic theory, but through probability analysis of economic time series itself or random nature, let the data speak for themselves, in order to find the inherent law. The historical information of time series contains its own law of fluctuation, so it can be used to interpret and predict the series of historical information.

CPI time series has a self-driven internal dynamic mechanism, so that the fluctuation of time series follows a specific law. This article will use the CPI monthly data from January 2008 to December 2016 to describe the internal driving mechanism of CPI, and predict CPI on this basis.

\section{Time Series Feature Analysis}

The first adjustment for the January 2008 to December 2016 monthly log data from CPI, and the treatment of the drawing data analysis found that in early 2009 there is a clear downward trend and has a clear upward trend, in the end it seems intuitive, LnCPI is a significant non-stationary sequence. In order to reduce the volatility, we make the first order difference and plot it to find that the difference sequence is far from the original sequence, so we intuitively believe that the LnCPI sequence is stable after the first order difference. To further validate our view, we tested the ADF unit root for the first difference LnCPI data, as shown in figure 1.

\begin{tabular}{cccc}
\hline \hline & & t-Statistic & Prob. $^{*}$ \\
\hline \hline \multicolumn{2}{c}{ Augmented Dickey-Fuller test statistic } & -4.897773 & 0.0001 \\
\hline Test critical & & & \\
values: & $1 \%$ level & -3.493747 & \\
& $5 \%$ level & -2.889200 & \\
& $10 \%$ level & -2.581596 & \\
\hline \hline
\end{tabular}

Figure 1 ADF unit root test 
As can be seen from the table, the ADF test results are -4.897773 , smaller than the -3.493747 at the 0.01 level, which is significant at the level of $1 \%$, so it is reasonable to conclude that the first order difference sequence of LnCPI is a stationary sequence.

\section{Model Parameter Estimation}

We do correlation diagram for the data sequence, found from the autocorrelation function of the data and the partial autocorrelation function graph we can, in the autocorrelation function, from second order to fourth order significantly, from the beginning of the fifth order, significantly decreased; in the partial autocorrelation function in order from second to sixth order are more significant, from the beginning of the seventh order, significantly decreased. Therefore, according to the above analysis results, the initial set of $\mathrm{p}=2, \mathrm{q}=6$.

The model ARMA $(2,6)$ can be initially established according to the above model's initial order. In order to predict and analyze the model better and more accurately, it is necessary to try different model fitting, such as ARMA (1,6), ARMA (2,5) and ARMA (1,5). In the various model parameters on the significant test, only ARMA $(1,4)$ and ARMA $(1,6)$ of all the parameters in the two models are significant, so further, according to the relevant information criterion of these two models to the final model selection.

Table 1 Model related information criteria

\begin{tabular}{|c|c|c|c|}
\hline Model & AIC & SC & HQC \\
\hline ARMA(1,6) & $-7,707,254$ & -7.581620 & -7.656334 \\
\hline ARMA(1,4) & -7.662922 & -7.485991 & -7.591226 \\
\hline
\end{tabular}

From table 1 shows, through the comparison of the two models show three different information criteria, either by which information criterion (1,6), ARMA's information criterion values are less than the corresponding ARMA $(1,4)$ value, so, finally we choose the ARMA $(1,6)$ model, the model estimation the regression results are as follows:

$$
\begin{aligned}
& \widehat{X}_{\mathrm{t}}=-0.000239+0.0763357 \mathrm{X}_{\mathrm{t}-1}-1.053283 \alpha_{\mathrm{t}-1}+0.515013 \alpha_{\mathrm{t}-2}+0.345885 \alpha_{\mathrm{t}-6} \\
& \begin{array}{lllll}
(-0.145063) & (9.762499) & (-11.33264) & (7.342436) & (6.621474)
\end{array}
\end{aligned}
$$

The error variance estimate is: $\hat{\sigma}_{\alpha}=0.005014$

\section{Adaptive Test of the Model}

After the parameter estimation, the adaptability of the fitting model is tested, in essence, the model residual sequence is white noise test. If the residuals are not white noise, there are still some important information in the model, and the model should be re established without being extracted.

First, the residuals of the model are extracted from 2008 to 2016, and the results are as figure 2. From the (partial) correlation function value, Q-Stat and the $\mathrm{p}$ value showed that the residual sequence does not exist autocorrelation, white noise, the actual value of the effect is good, therefore, we believe that with the fitted values, the established model is appropriate. 


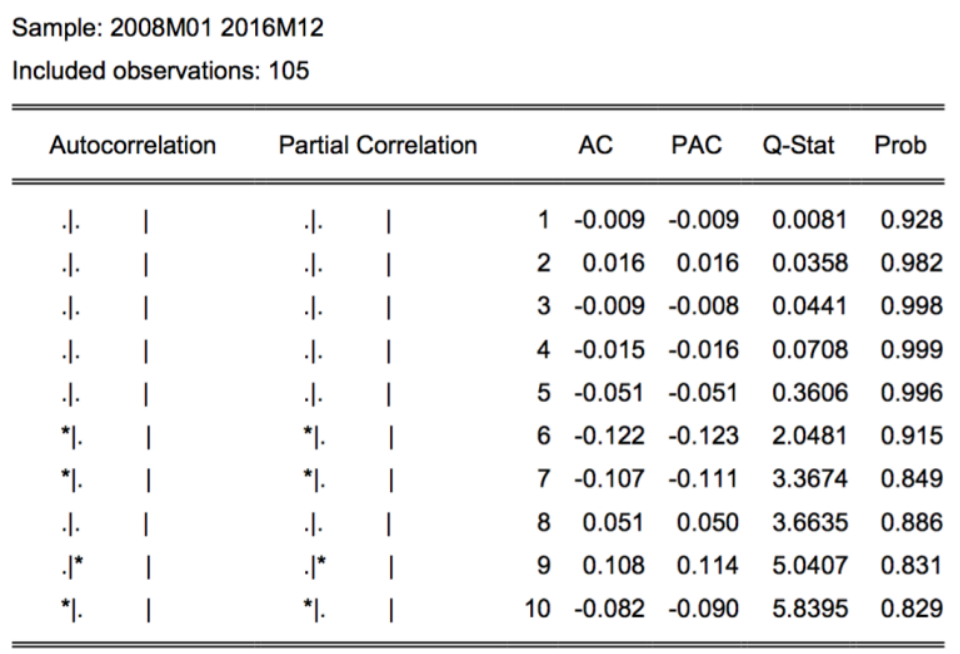

Figure 2 White noise test of the model

\section{Model prediction results}

The use of ARIMA $(1,1,6)$ model for static prediction of the CPI trend forecast period from January 2008 to December 2016, from the view of the expected results, the model has good prediction effect, error rate is 0.00103 , the standard deviation is 0.004055 (as shown in Figure 3 ), it shows that the prediction accuracy of this regression is relatively satisfactory.
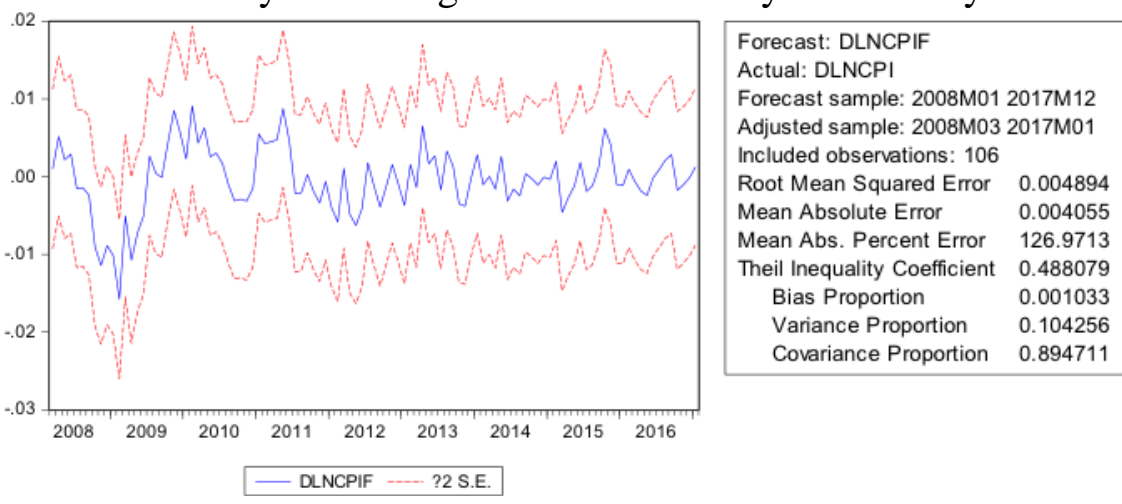

Figure 3 Static prediction of CPI in 2008-2016

At this point, you can extend the sample period and predict the CPI for January 2017. According to the model, the CPI value in 2017 is 102.38 , the actual value is 102.5 , from the prediction results, the ARIMA prediction model has a good effect on CPI prediction. The sample period is progressively expanded, and the parameters of the ARIMA model are re estimated, and further predictions are made. The prediction results are shown in Table 2 below.

Table 2 Static prediction of CPI in 2007

\begin{tabular}{|c|c|c|c|c|c|c|}
\hline Time & Jan & Feb & Mar & Apr & May & Jun \\
\hline $\begin{array}{c}\text { Predicted } \\
\text { Value }\end{array}$ & 102.38 & 101.02 & 101.08 & 101.23 & 102.1 & 102.14 \\
\hline Time & Jul & Aug & Sep & Oct & Nov & Dec \\
\hline $\begin{array}{c}\text { Predicted } \\
\text { Value }\end{array}$ & 102.14 & 102.08 & 102.07 & 102.07 & 101.85 & 101.76 \\
\hline
\end{tabular}

\section{References}

[1] Guo Xiaofeng. Prediction and analysis of CPI trend in China based on ARIMA model. [J].Statistics and Decision, 2012 (11): 29-32.

[2] Yi Danhui. Data analysis and EViews application.[M]Renmin University of China press, 2008. 
[3] Geng Juanjuan, Sun Jufang. Based on time series analysis, the trend of the stock index of Shanghai stock market.[J]. Management Observation, 2014 (29): 96-97.

[4] Dong Mei. Prediction of consumer price index based on VAR model. [J]. Statistics and Decision, 2011 (1): 29-31.

[5] Li Feiao, Luo Wenqiang, Liu Xiaoshan, et al. Study on prediction of landslide deformation by multivariate non-stationary time series [J]. Journal of Yangtze Academy of Sciences, 2014, 31 (4): 31-34.

[6] Huang Yong. Study on the time series of China's consumer price index [J]. modern business and trade industry, 2011, 23 (21): 7-8.

[7] Wang Shaoping, Zhu Manzhou, Hu Shuo. The macro component and macro impact of Chinese CPI. [J]. Economic Research, 2012 (12): 29-42.

[8] Wang Chunying, Li Fei, Wang Huizheng. An empirical analysis of CPI short term forecasting in China. Based on the perspective of CPI, [J]. Thought Front, 2013 (S1): 101-103.

[9] Han Chunlei, Wan Jun high. Our monthly CPI combined forecast and [J]. Statistical Analysis and Decision Making, 2014, 28 (1): 11-13. 